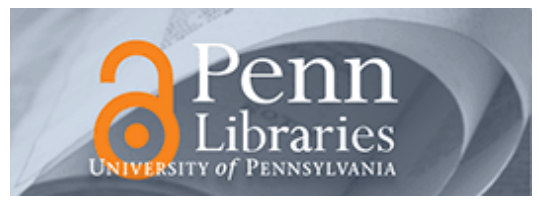

University of Pennsylvania

ScholarlyCommons

Statistics Papers

Wharton Faculty Research

$5-1985$

\title{
A Stochastic Model of Fragmentation in Dynamic Storage
} Allocation

\author{
E. G. Coffman Jr
}

T. T. Kadota

Larry A. Shepp

University of Pennsylvania

Follow this and additional works at: https://repository.upenn.edu/statistics_papers

Part of the Computer Sciences Commons, and the Statistics and Probability Commons

\section{Recommended Citation}

Coffman, E. G., Kadota, T. T., \& Shepp, L. A. (1985). A Stochastic Model of Fragmentation in Dynamic Storage Allocation. SIAM Journal on Computing, 14 (2), 416-425. http://dx.doi.org/10.1137/0214032

This paper is posted at ScholarlyCommons. https://repository.upenn.edu/statistics_papers/408

For more information, please contact repository@pobox.upenn.edu. 


\title{
A Stochastic Model of Fragmentation in Dynamic Storage Allocation
}

\author{
Abstract \\ delay with rate parameter $\mu$, after which the location again becomes available. The set of occupied \\ by independent arguments we show that $(E \max (S)-E|S|) / E|S| \rightarrow 0$ as the traffic intensity $\rho \rightarrow \infty$. \\ never more than $1 / 3$ the expected number of occupied locations. \\ Our model applies to studies of fragmentation in paged computer systems, and to containerization \\ problems in industrial storage applications. Finally, our model can be regarded as a simple concrete \\ model of interacting particles [Adv. Math., 5 (1970), pp. 246-290].

\section{Keywords} \\ dynamic storage allocation, checkerboarding, $\mathrm{M} / \mathrm{M} / \infty$ queue, memory allocation \\ Disciplines \\ Computer Sciences | Statistics and Probability
}

We study a model of dynamic storage allocation in which requests for single units of memory arrive in a Poisson stream at rate $\lambda$ and are accommodated by the first available location found in a linear scan of memory. Immediately after this first-fit assignment, an occupied location commences an exponential locations (identified by their numbers) at time $t$ forms a random subset $S_{t}$ of $\{1,2, \ldots\}$. The extent of the fragmentation in $S_{t}$, i.e. the alternating holes and occupied regions of memory, is measured by $\left(S_{t}\right)-\left|S_{t}\right|$. In equilibrium, the number of occupied locations, $|S|$, is known to be Poisson distributed with mean $\rho=\lambda /$ $\mu$. We obtain an explicit formula for the stationary distribution of max (S), the last occupied location, and Moreover, we verify numerically that for any $\rho$ the expected number of wasted locations in equilibrium is 


\title{
A STOCHASTIC MODEL OF FRAGMENTATION IN DYNAMIC STORAGE ALLOCATION*
}

\author{
E. G. COFFMAN, JR. $\dagger^{\dagger}$, T. T. KADOTA $\dagger$ AND L. A. SHEPP $\dagger$
}

\begin{abstract}
We study a model of dynamic storage allocation in which requests for single units of memory arrive in a Poisson stream at rate $\lambda$ and are accommodated by the first available location found in a linear scan of memory. Immediately after this first-fit assignment, an occupied location commences an exponential delay with rate parameter $\mu$, after which the location again becomes available. The set of occupied locations (identified by their numbers) at time $t$ forms a random subset $S_{t}$ of $\{1,2, \cdots\}$. The extent of the fragmentation in $S_{t}$, i.e. the alternating holes and occupied regions of memory, is measured by $\max \left(S_{t}\right)-\left|S_{t}\right|$. In equilibrium, the number of occupied locations, $|S|$, is known to be Poisson distributed with mean $\rho=\lambda / \mu$. We obtain an explicit formula for the stationary distribution of $\max (S)$, the last occupied location, and by independent arguments we show that $(E \max (S)-E|S|) / E|S| \rightarrow 0$ as the traffic intensity $\rho \rightarrow \infty$. Moreover, we verify numerically that for any $\rho$ the expected number of wasted locations in equilibrium is never more than $\frac{1}{3}$ the expected number of occupied locations.

Our model applies to studies of fragmentation in paged computer systems, and to containerization problems in industrial storage applications. Finally, our model can be regarded as a simple concrete model of interacting particles [Adv. Math., 5(1970), pp. 246-290].
\end{abstract}

Key words. dynamic storage allocation, checkerboarding, $M / M / \infty$ queue, memory allocation

1. Introduction. Adopting the terminology of queues, suppose customers arrive in a Poisson stream at rate $\lambda$ to a linear queue of waiting or storage locations numbered $1,2, \cdots$. According to the so-called first-fit policy each customer occupies the lowest numbered location available at his time of arrival. Immediately upon being installed in an available location, a customer commences a delay or residence time having an exponential distribution with parameter $\mu$. At the end of his residence time a customer departs from the queue, thus making available the location he occupied. As locations are occupied and released "holes" build up, so that the total occupancy, defined as the highest numbered location occupied by waiting customers, may be substantially greater than the number of customers in the queue. The principal objectives of this paper are an analysis leading to the stationary distribution of this total occupancy, and a characterization of the fraction of wasted space under heavy-traffic conditions, i.e. for large $\lambda / \mu$.

In queueing parlance our model may be recognized as an $M / M / \infty$ queue on which a first-fit discipline for placement into a linear sequence of servers has been superimposed. The equilibrium distribution for the number in system is well-known for the $M / M / \infty$ system $[4$, p. 414].

Although we shall make some use of these classical results, we focus on the more difficult analysis of the total occupancy process, an analysis that is clearly more important in the applications noted below. Similar results for an $M / M / 1$ queue have been obtained in [2], where conventional methods were found to be adequate. The greater difficulty of our problem stems from the more easily motivated, but combinatorially more complex, first-fit placement rule.

Interpreting customers as requests for single units of storage, our model is an instance of the general problem of dynamic storage allocation in computers. The elements of this subject have been treated by Knuth [6], and a recent survey appears in [1]. In particular, the infinite-server model was introduced in [6, p. 445] in an analysis of the well-known fifty-percent rule.

* Received by the editors September 28, 1982, and in revised form January 30, 1984.

$\dagger$ AT\&T Bell Laboratories, Murray Hill, New Jersey 07974. 
Paged computer systems are specific applications of our model. Here, single units of storage become pages and locations become page frames, i.e. sets of consecutive memory locations that can accommodate exactly one page and that begin at integral multiples of the fixed page size. As before, the analysis of total occupancy leads to a characterization of the extent of fragmentation that occurs as pages come and go under a first-fit rule. Indeed, by an appropriate substitution of terms, our model applies quite generally to any such first-fit storage/server assignment problem where locations would correspond, for example, to telephone trunks, parking spaces, etc. There are a number of extensions to our model which would broaden its applicability. These, along with their implications for the analysis of stochastic models, are discussed in the last section.

Our analysis starts with the observation that the total occupancy process cannot be formulated as a Markov chain. We then identify a bivariate Markov chain in continuous time from which the stationary state probabilities of this process can be calculated. This calculation amounts primarily to a solution of the partial differential equation governing the generating function for the equilibrium probabilities of the bivariate Markov chain. For this purpose we adopt an apparently novel approach with generating functions, whereby a partial differential equation is replaced by an infinite but solvable system of ordinary differential equations. By an independent argument bounds on the expected total occupancy are derived; asymptotic properties of the total occupancy process are deduced from these bounds.

In the next section the mathematical model is formally defined. The major results, also presented in the next section, are then proved in $\S \S 3$ and 4 .

2. Mathematical model. For a fixed traffic intensity $\rho=\lambda / \mu>0$ consider a continuous time Markov chain $M_{\rho}$ whose states are the (finite) subsets of $\{1,2, \cdots\}$. A given state $S_{t}$ is just that collection of numbers corresponding to occupied locations at time $t$. Transitions occur at rate $\mu>0$ from any nonempty $S$ to each of the $|S|$ subsets of $S$ obtained by deleting one location from $S$, and at rate $\lambda$ from any $S$ to the union of $S$ and the smallest numbered location not in $S$. Because $S$ diminishes at rate $|S| \mu$, it is easy to see that for any $\rho$, if $\left|S_{0}\right|<\infty$ then $\left|S_{t}\right|<\infty$ for all $t>0$ with probability 1 and that $M_{\rho}$ is a Markov chain with a stationary distribution on the set of finite subsets of $\{1,2, \cdots\}$. It does not seem possible to obtain the stationary distribution of $S$, but we are able to find this distribution for both $|S|$ and $\max (S)$, the maximum element of $S$. Note that $\max (S)$ is simply the total occupancy mentioned in the previous section.

Letting $\pi_{k}=\lim _{t \rightarrow \infty} P\left(\left|S_{t}\right|=k\right), k=0,1, \cdots$, denote the stationary distribution, we have the following standard results from the analysis of an $M / M / \infty$ queue [4]

$$
\pi_{k}=\frac{\rho^{k}}{k !} e^{-\rho}, \quad k=0,1, \cdots
$$

and

$$
E|S|=\sum_{k \geqq 0} k \pi_{k}=\rho .
$$

Next, consider the distribution of $\max (S)$. We will prove in $\S 3$ that

$$
P(\max (S)>m)=\sum_{n=0}^{\infty} \frac{(-1)^{n}}{n+1} \frac{1}{\sum_{k=0}^{m}\left(\begin{array}{l}
m \\
k
\end{array}\right)(n+k) ! / \rho^{n+k+1}}, \quad m=0,1, \cdots .
$$

Note that for $m=0, P(\max (S)>0)=1-P(S=\varnothing)=1-P(|S|=0)=1-e^{-\rho}$, as seen from (2.1), and (2.3) agrees with this result. For $m=1$, the right side of (2.3) becomes 
an integral and

$$
P(\max (S)>1)=1-\int_{0}^{1} e^{-\rho x^{1 / \rho}} d x .
$$

However, for large $m$ and $\rho$, calculation of the right side of (2.3) is awkward. For these cases we can make use of the following crude bounds,

$$
1-\sigma_{m} \leqq P(\max (S)>m) \leqq \rho \frac{\sigma_{m}-\sigma_{m-1}}{\sigma_{m}},
$$

where

$$
\sigma_{m}=e^{-\rho} \sum_{k=0}^{m} \frac{\rho^{k}}{k !}, \quad m=0,1, \cdots
$$

To prove $(2.4)$, note that $\max (S) \geqq|S|$ so that $P(\max (S)>m) \geqq P(|S|>m)$; the first inequality thus follows from (2.1) and (2.5). For the second, note first that

$$
P(\max (S)>m) \leqq E|S \cap\{m+1, m+2, \cdots\}|=E|S|-E|S \cap\{1,2, \cdots, m\}| .
$$

Now observe that $\left|S_{t} \cap\{1, \cdots, m\}\right|$ is an embedded Markov process on states $0,1, \cdots, m$, and that its stationary distribution can be easily obtained as

$$
P(|S \cap\{1, \cdots, m\}|=k)=\frac{\rho^{k}}{k !} \frac{1}{\sum_{j=0}^{m} \rho^{j} / j !}, \quad k=0,1, \cdots, m .
$$

Using (2.7) in (2.6) gives the second inequality in (2.4).

We can use (2.4) to show that the rate of wastage (the fraction of holes) is asympotically negligible for $\rho \rightarrow \infty$. First note that, for any integer $K$,

$$
E \max (S)=\sum_{m=0}^{\infty} P(\max (S)>m) \leqq K+\sum_{m=K}^{\infty} P(\max (S)>m) .
$$

Choosing $K=\lfloor(1+\varepsilon) \rho\rfloor$ and using (2.4) and (2.8), we have, since $\sigma_{m} \uparrow 1$,

$$
\frac{E \max (S)}{\rho} \leqq 1+\varepsilon+\frac{1-\sigma_{K-1}}{\sigma_{K}} \text {. }
$$

Since for every $\varepsilon>0,1-\sigma_{K-1} \rightarrow 0$ as $\rho \rightarrow \infty,[4$, p. 193] it follows from (2.9) that

$$
\varlimsup_{\rho \rightarrow \infty} \frac{E \max (S)}{\rho} \leqq 1 .
$$

On the other hand, $\max (S) \geqq|S|$, thus $E \max (S) \geqq E|S|=\rho$. Hence

$$
E(\max (S)-|S|)=o(E|S|) \quad \text { as } \rho \rightarrow \infty .
$$

Thus, as $\rho \rightarrow \infty$ the wastage becomes negligible relative to the number of occupied locations.

The method of (2.8) can be used to tighten the bound of (2.9) on $E \max (S)$ by applying (2.8) for $K=\left\lfloor(\rho \log \rho)^{1 / 2}\right\rfloor$ instead of $K=\lfloor(1+\varepsilon) \rho\rfloor$. In fact we show in $\S 4$ that

$$
E(\max (S)-|S|) \leqq c(\rho \log \rho)^{1 / 2}
$$

for some constant $c$. For an even closer look at asymptotic behavior numerical calculations were worked out by using (2.3) directly in (2.8). A plot of $\log P(\max (S)>m)$ 
vs. $\log 1 / \rho$ revealed a nearly linear graph and thus indicated that, as $\rho \rightarrow \infty$

$$
E(\max (S)-|S|) \sim c^{\prime} \rho^{\alpha},
$$

for constants $\alpha$ and $c^{\prime}$. The numerical results showed that $\alpha \approx 0.42$.

In Fig. 1, the wastage rate, defined as $(E \max (S)-E|S|) / E|S|$, is plotted as a function of $1 / E|S|=1 / \rho$. It shows that the wastage rate approaches 0 monotonically as $\rho \rightarrow \infty$ and $\rho \rightarrow 0$. What is more interesting, however, is the existence of a maximum clearly less than $1 / 3$ at $1 / \rho=0.40$ and the strictly monotone behavior on both sides of the maximum. Thus, $E \max (S)$ is at most $\frac{4}{3}$ times $E|S|$.

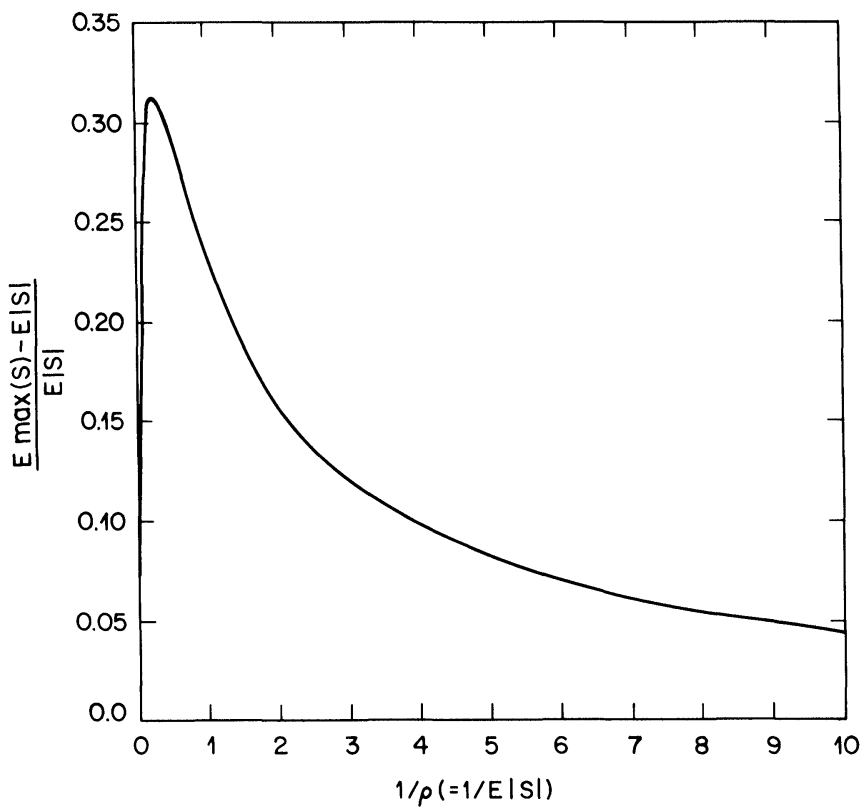

FIG. 1.

3. Derivation of (2.3). The derivation depends first on the use of an embedded two-dimensional Markov process and then on the use of a novel technique with generating functions. The embedded Markov process is $\left(\left|S_{t} \cap\{1,2, \cdots, m\}\right|, \mid S_{t} \cap\right.$ $\{m+1, m+2, \cdots\} \mid)$ which is Markovian on states of integer pairs $(k, r), 0 \leqq k \leqq m, 0 \leqq r$. The stationary distribution, for fixed $m \geqq 0$,

$$
\pi(k, r)=\pi(k, r ; m)=P(|S \cap\{1, \cdots, m\}|=k,|S \cap\{m+1, m+2, \cdots\}|=r)
$$

satisfies the equations

$$
\pi(k, r)(\lambda+(k+r) \mu)=\pi(k-1, r) \lambda+\pi(k+1, r)(k+1) \mu+\pi(k, r+1)(r+1) \mu
$$

for $0 \leqq k<m, 0 \leqq r$ where $\pi(-1, r)=0$, and

$$
\pi(m, r)(\lambda+(m+r) \mu)=\pi(m-1, r) \lambda+\pi(m, r-1) \lambda+\pi(m, r+1)(r+1) \mu
$$

for $r \geqq 0$, where $\pi(m,-1)=0$. Introducing the generating function

$$
F(x, y)=\sum_{k=0}^{m} \sum_{r=0}^{\infty} \pi(k, r) x^{k} y^{r}
$$


and $\rho=\lambda / \mu$, we obtain from (3.2) and (3.3)

$$
\rho(1-x) F(x, y)+(x-1) \frac{\partial F}{\partial x}(x, y)+(y-1) \frac{\partial F}{\partial y}(x, y)=\rho x^{m}(y-x) f_{m}(y)
$$

where

$$
f_{m}(y)=\sum_{r=0}^{\infty} \pi(m, r) y^{r}=\frac{1}{m !} \frac{\partial^{m}}{\partial x^{m}} F(x, y) .
$$

In view of the fact that

$$
P(\max (S) \leqq m)=P(|S \cap\{m+1, m+2, \cdots\}|=0)=\sum_{k=0}^{m} \pi(k, 0)=F(1,0),
$$

we want to find $F(1,0)$. The difficulty with solving (3.5) for $F$ is that it is a partial differential equation (and, moreover, of degree $m$ by (3.6)). However, by alternately setting $x=1$ and $y=1$ and repeatedly differentiating, we can reduce (3.5) to an infinite series of ordinary differential equations which we can solve.

First, setting $x=1$ in $(3.5)$ and noting that $F(1,1)=1$, we obtain

$$
F(1, y)=1-\rho \int_{y}^{1} f_{m}(u) d u .
$$

Next, setting $y=1$ in (3.5), we obtain

$$
F(x, 1)-\frac{1}{\rho} \frac{\partial F}{\partial x}(x, 1)=x^{m} f_{m}(1),
$$

and solving this (ordinary) differential equation, with $F(1,1)=1$,

$$
F(x, 1)=\rho e^{\rho x} \int_{x}^{\infty} e^{-\rho u} u^{m} d u f_{m}(1)
$$

Now, for each $n \geqq 1$, differentiate (3.5) $n$ times with respect to $y$ and then set $y=1$, to obtain

$$
\begin{aligned}
\rho(1-x) D_{y}^{n} F(x, 1) & +(x-1) \frac{\partial}{\partial x}\left[D_{y}^{n} F(x, 1)\right]+n D_{y}^{n} F(x, 1) \\
& =\rho x^{m}(1-x) f_{m}^{(n)}(1)+n \rho x^{m} f_{m}^{(n-1)}(1)
\end{aligned}
$$

where $D_{y}^{n}=\partial^{n} / \partial y^{n}$ and

$$
f_{m}^{(n)}(y)=D_{y}^{n} f_{m}(y), \quad n=0,1,2, \cdots .
$$

Solving (3.11) for $D_{y}^{n} F(x, 1)$ we obtain

$$
\begin{array}{r}
D_{y}^{n} F(x, 1)=-\frac{1}{(x-1)^{n}} \rho e^{\rho x} \int_{x}^{\infty} d u e^{-\rho u}(u-1)^{n-1}\left[u^{m}(1-u) f_{m}^{(n)}(1)+n u_{m}^{m} f_{m}^{(n-1)}(1)\right], \\
n=1,2, \cdots .
\end{array}
$$

Since $F(x, y)$ is a polynomial in $x$ for any $y$, the right side of (3.13) must be finite at $x=1$ and hence the integral in (3.13) must vanish at $x=1$, namely,

$$
\int_{1}^{\infty} d u e^{-\rho u}(u-1)^{n-1}\left[u^{m}(1-u) f_{m}^{(n)}(1)+n u^{m} f_{m}^{(n-1)}(1)\right]=0, \quad n \geqq 1
$$


Rewriting (3.14),

$$
\frac{f_{m}^{(n)}(1)}{n !} \int_{1}^{\infty} d u e^{-\rho u}(u-1)^{n} u^{m}=\frac{f_{m}^{(n-1)}(1)}{(n-1) !} \int_{1}^{\infty} d u e^{-\rho u}(u-1)^{n-1} u^{m}, \quad n \geqq 1,
$$

we observe that the function described by the left side of (3.15) does not depend on $n=0,1,2, \cdots$. Setting $n=1$ in the right side of (3.15), then using (3.10) and the fact that $F(1,1)=1$, we have

$$
\frac{f_{m}^{(n)}(1)}{n !} \int_{1}^{\infty} d u e^{-\rho u}(u-1)^{n} u^{m}=\frac{f_{m}^{(0)}(1)}{0 !} \int_{1}^{\infty} d u e^{-\rho u} u^{m}=\frac{e^{-\rho}}{\rho}
$$

Now, using Taylor's expansion about $y=1$,

$$
f_{m}(y)=\sum_{n=0}^{\infty} \frac{f_{m}^{(n)}(1)(y-1)^{n}}{n !} .
$$

Since the coefficients $\pi(m, r)$ in (3.6) satisfy $\pi(m, r) \leqq P(|S| \geqq m+r)=O(1 / r !)$ by (2.1), $f_{m}$ is an entire function and the validity of (3.17) follows. Using (3.16) and (3.17), we have

$$
f_{m}(y)=\frac{e^{-\rho}}{\rho} \sum_{n=0}^{\infty}(y-1)^{n} \frac{1}{\int_{1}^{\infty} d u e^{-\rho u}(u-1)^{n} u^{m}} .
$$

Then, from (3.8),

$$
F(1, y)=1-e^{-\rho} \sum_{n=0}^{\infty} \frac{(y-1)^{n+1}}{(n+1)} \frac{1}{\int_{1}^{\infty} d u e^{-\rho u}(u-1)^{n} u^{m}}
$$

and setting $y=0$ gives (2.3) because of (3.7).

We observe finally that the joint distribution of $\max (S)$ and $|S|$ can be obtained from $F(x, y)$, which is determined from (3.13) and (3.16) explicitly. Indeed

$$
P(\max (S) \leqq m,|S| \leqq k)=\sum_{j=0}^{k} \pi(j, 0 ; m)
$$

and $\pi(j, 0 ; m)=\pi(j, 0)=\partial^{j} / \partial x^{j} F(0,0)$, which can be obtained from $F(x, y)$. The resulting expression for the joint distribution of $\max (S)$ and $|S|$ does not appear to have a simple form and is therefore omitted.

4. Derivation of (2.12). First, note that

$$
\sum_{m=K}^{\infty} \frac{x^{m}}{m !} \leqq \frac{x^{K}}{K !}\left(1+\frac{x}{K}+\left(\frac{x}{K}\right)^{2}+\cdots\right)=\frac{x^{K}}{K !} \frac{K}{K-x}, \quad x>0 .
$$

From (2.8) and (2.4) with $\rho=x$

$$
E \max (S) \leqq K+x \sum_{m=K}^{\infty} \frac{x^{m}}{m !} \frac{1}{\sum_{j=0}^{m}\left(x^{j} / j !\right)} .
$$

Since $e^{-x} \sum_{j=0}^{\lfloor x\rfloor}\left(x^{j} / j !\right) \rightarrow \frac{1}{2}$ as $x \rightarrow \infty[4$, p. 193], by setting $K=\lfloor x+y\rfloor$ we obtain, using (4.1) with Stirling's formula,

$$
\begin{aligned}
E \max (S) & \leqq x+y+c_{1} x e^{-x} \frac{x^{x+y}}{(x+y)^{1 / 2}(x+y)^{x+y}} e^{-(x+y)} \frac{x+y}{y} \\
& \leqq x+y+\frac{c_{1}(x+y)^{3 / 2} e^{y}}{y \exp [(x+y) \log (1+y / x)]} \\
& \leqq x+y+\frac{c_{1}(x+y)^{3 / 2} e^{y}}{y \exp \left(y+y^{2} / 2 x\right)}
\end{aligned}
$$


for an appropriate constant $c_{1}>0$. Then, setting $x=\rho$ and $y=c_{1}(\rho \log \rho)^{1 / 2}$, we have

$$
E \max (S) \leqq \rho+c_{1}(\rho \log \rho)^{1 / 2}+\frac{\left[\rho+c_{1}(\rho \log \rho)^{1 / 2}\right]^{3 / 2}}{(\rho \log \rho)^{1 / 2} \rho^{c_{1}^{2 / 2}}}
$$

Thus, by choosing $c_{1}$ appropriately, say $c_{1}=(2+\varepsilon)^{1 / 2}$, the last term of (4.4) can be made to vanish as $\rho \rightarrow \infty$. Finally, by modifying the constant of the second term of (4.4), we obtain (2.12).

5. Discussion. The main results of this paper have been the explicit form in (2.3) for the stationary distribution of $\max (S)$, and the asymptotic behavior of the wastage rate given by (2.11). Two principal conclusions concerning fragmentation are that the stationary expected total occupancy, $E \max (S)$, never exceeds about $\frac{4}{3}$ the minimum possible expectation, $E|S|$, and that the difference between the two is asymptotically $o(E|S|)$; i.e. for sufficiently heavy traffic the effects of fragmentation become unimportant. Although proved only for the first-fit rule, one would expect this asymptotic behavior of any rule that places newcomers, whenever possible, in an unoccupied location less than $\max \left(S_{t}\right)$.

We have considered several extensions to the basic model with the same objectives in each case: an explicit form for $P(\max (S)=m)$ in equilibrium and a characterization of the asymptotic wastage rate. While the first objective appears unattainable (and hence not treated in detail here), these extensions are given below as open problems, since the prospects for asymptotic results or easily computed numerical results may be much better.

1. Extensions to the delay $(M / M / \infty)$ queue. Suppose there is a fixed bound, $M$, on the number of locations: arrivals at times $t$ when $\left|S_{t}\right|=\max \left(S_{t}\right)=M$ are simply lost. Our basic process $\left[S_{t} \cap\{1,2, \cdots, m\}, S_{t} \cap\{m+1, \cdots, M\}\right]$ is now a bivariate finite Markov chain. Although explicit forms are apparently limited to matrix equations, conventional numerical methods can be employed to study the influence of the boundary. The results of preceding sections serve as descriptions of the asymptotic behavior, $M \rightarrow \infty$.

As another extension to the original model, suppose all arrivals now request a fixed number $l>1$ of consecutive locations, and are accommodated by a first-fit policy; departures occur individually as before. It is easy to verify for this bulk-arrival queue that the process $\left[S_{t} \cap\{1,2, \cdots, m\}, S_{t} \cap\{m+1, m+2, \cdots\}\right]$ no longer has the Markov property. Indeed, the types of fragmentation now possible are such that any Markov chain from which $P\left(\max \left(S_{t}\right)=m\right)$ could be calculated would have a much larger state space (essentially, it is now necessary to consider ordered subsets).

It is also interesting to question whether the wastage rate is still asymptotically negligible in this model. In contrast to the $l=1$ case it is now possible for $\max \left(S_{t}\right)$ to increase even though holes exist. Equivalently, $\max \left(S_{t}\right) \leqq \max _{\tau \leqq t}\left|S_{\tau}\right|$ no longer holds for $l>1$. It would be especially interesting to resolve this issue analytically in the general model where $l$ is a random variable with a stationary distribution, $\left\{f_{i}\right\}$, and all locations of a given request are made available (i.e. depart) at the same instant. For if $E \max (S)-E|S|$ were not asymptotically $o(E|S|)$ as $\rho \rightarrow \infty$ then this would be further strong support for the concept of paging (or containerization in a general industrial setting).

To acquire further insight into this general problem, a number of simulations were run on first-order extensions of the basic model. First, request sizes were allowed to be either 1 or 2 locations and second, either 2 or 3 locations. In addition to the parameters $\lambda$ and $\mu$ we have the parameter $p$ denoting the stationary probability that a given request is for the smaller of the two sizes. 
Typical results of these simulations are shown in the checkerboard patterns of Fig. 2, where locations $1-100$ are in row 1 of the tableaus, locations 101-200 in row 2, etc. An integer $k$ in some location of a tableau signifies that this location is one of a sequence of $k$ allocated to a single request ( $k=0$ denotes an available location). For

11111111111111111111111111111111111111111111111111111111111111111111111111111111111111111111111111111 111111111111111111111111111111111111111111111111111111111111111111111111111111111111111111111111111111 111111111111111111111111111111111111111111111111111111111111111111111111111111111111111111111111111 11111111111111111111111111111111111111111111111111111111101111111111111111111111111111111111111111 1111111111111111111111111111111111111111111111111111111111111111111111111111111111111111111111111111 11111111111111111111111111111111111111111111111111111111111111111111111111111111111111111111111111111 111111111111111111111111111111111111111111111111111111111111111111111111111111111111111111111111111 111111111111111111111111111111111111111111111111111111111111111011111111111111111111111111111111111111 11111111111111111111111111111111111111110111111111111111111111111111111111111111111111111111111111 1111011101111111111011111111111111111111111111111011111111111111111111111110001101111111000111101010 111100100101010001011

$$
\text { a. } \lambda=1, \mu=0.001
$$

11111111111111111111111111111111111111111111111111111111111111111111111111111111111111111111221111 111111111111112211111111111111122111111111111221111111111111111111111111111111112211111111122111111 111111111111111111111111111111112211111111111111111111111111111122111111111111111111111111111111111 111111111111111111111111111111111111111111111111111122111111111111111111111111111111111111112211221 1111111111111122122111111111111112212211112211101221221122122222222221112222111111111112211111221221 12222122122221111221222212212222221222212222111111222222212211122222212222221222212211112212222222222 1220222212222221221122122222222012222222212222222222122012222222202222022222222222222222211122221222 22222222222222222221222222122222222222202222221022222222111221122222222222222222222222222222222222222 22022222222222222222222222202222222222022222202222022222222222222222222222222222022222222222222222222 22222212222222202222222222222222222222220222222222222222222222222202222222222222222222222222222222222 22222222222222222222222222222222222222222222222222222222222222222222222222222222222222222222222222222 22222222222222222222222222222222002222222222222222222222222222222222222222222222222222222222222222200 22222222222222222222222222222222222222222202202222222222222222222222222222222222222222222222222222222 22222222222222222222222222222222222222222222222222222222222222222222222222222222222222222002222222222 22222222222222222222222222222222000022222222000022222222200222222222222222200222200222222222222222222 22222222222222222200000022002200002200002200220022

$$
\text { b. } \lambda=1, \mu=0.001, p=1 / 2
$$

2222333222233322222222022333333333222222222222222222222222222222222222333222222222222222222222233322 22222222222222022222233322222222220222222222222222222222223332222333222222222233322222222222222333222 22222333222222222222222222333222222220222222222202222222222222222222222222222222333220222233322222222 22222222222222223332222333222222222233322222222333322222222022222222222222222222222222222222222222223 33333333220333222222223332222222222222222333222233332222333222222220222222333222222222222222222223332 222222022233322222222203332233322222222333222222222222233322333222222222233322222222333222222220222233 33332222333222233322333222222222222222233322333222222333322333222222223332233322222222222203332222222 22222220222233322220333222222022333222233333322222222222022222222333222222222222333333220223333332222 22222233322220222222223333332202222222222222222223333333222222222222222203333332233322223333332222223 332222333222222223333333223333332222222233322222222022333322223333332222222222222222220022222233322333 222233322222222022333223332222222222333223333333333333033333333333322333222233333332222333222222222233 333322333333222222333333333322333222222223333333332222333333322333333022322222222222233322222233322222 22222223333333332200223333333332233333333333322220223332222222222233333322333222233333322220022223333 3322222233333333303333333333333222200333222233303333332203333220033322333333333333333333322333333333333 3333332233333333322333333333333222333223333333332222222200333333322333333333222233303333333223333333333 33003333333333333330333333333333322333333223333333333330333333333333322333003332233333333332333333333333 32203332233333300333333333333333033333333333322223330333222233333333333330033333333322333223332233333 3033303333333332233300223330223333333333330333333003330333333333333333322333333333333333333333333333333 332233333333333333333333333333333333333333333333303333332233333330033322333333333333223333333333223333 333333333333333330033333333322333333333333333333333333333333333333333303333333333333333333333333333333 3333333330003333333333333333333333333330033333333333300333333333333333303333333333333333333333333333333 333333033333333333333333333333333333333330333333033333333333333333333333333333333333333333333333333333333 3333333333333333333333333333333333333330033333333333300333333333333300333333333333333333333333300333333 333033333333333333333333333333333333333333333333333333333333330033333333333303333333333333333333333333 0003333333333333333333333333333033333300033333300333333000333333333333333333300000033333333333330000003 333333333330003333333333333333333333330003333330003333333333333333333333333333333333333333333333333333 3000000333000000000333000333000000333000000333333000333

$$
\text { c. } \lambda=1, \mu=0.001, p=1 / 2
$$

FIG. 2. Storage patterns under heavy loading. 
comparison with our earlier analysis we have also shown results for the basic model having requests of size 1 only.

Figure $2 \mathrm{a}$ is consistent with our earlier asymptotic analysis in that the number of available locations is small relative to the number of occupied locations when $\rho$ is large $(\rho=\lambda / \mu=1000$ in the figure). As expected from a first-fit rule the available locations tend to be higher numbered ones.

In Fig. $2 b$ both of these effects are also illustrated for the system with two request sizes, 1 and 2, in spite of the greater fragmentation stemming from the fact that holes with a single location can be used only by requests for a single location. Accordingly, the first-fit rule justifies another obvious feature of the pattern, viz. that requests of size 1 tend to be concentrated in the smaller numbered locations.

The results from which Fig. $2 \mathrm{c}$ was selected also indicate that available locations tend to be higher numbered and smaller allocations tend toward the smaller numbers when request sizes are limited to 2 and 3 . On the other hand, fragmentation is somewhat worse in this system because of the occurrence of poor fits, e.g. a request of size 2 allocated the first two locations of a hole consisting of three available locations creates an unusable hole of one location. Thus, the above effects are somewhat less pronounced than in Fig. 2b.

Motivated by these limited simulations, the authors quite recently conducted much more extensive tests of asymptotic behavior. These simulation results (to appear in [2]) suggest strongly that our earlier conjecture in fact holds, i.e. first-fit is asymptotically optimal in the sense that $E \max (S)-E|S|=o(E|S|)$ as $\rho \rightarrow \infty$ for any distribution $\left\{f_{i}\right\}$. Another recent extension [5] of the present paper stems from the apparent difficulty in proving this conjecture, and is based on a modified first-fit rule. Although the new rule is unlikely to be selected in favor of the simple first-fit rule in practice, it is proved rigorously in [5], with the help of the results in this paper, that the new rule is asymptotically optimal.

2. Single server disciplines. Suppose we have the original first-fit model except that only one occupied location can be served (at rate $\mu$ ) at a time. In this case, of course, we need $\lambda<\mu$ for a stable system, i.e. for the existence of a stationary distribution. The preemptive, last-in-first-out service discipline, where the location being served is always max $\left(S_{t}\right)$, is trivially solved, for in this case $\left|S_{t}\right|$ and $\max \left(S_{t}\right)$ are identical and no fragmentation occurs. The standard solution for the stationary $M / M / 1$ queue applies.

Unfortunately, the more interesting first-in-first-out discipline suffers from the same general difficulties as the bulk-arrival $M / M / \infty$ queue described earlier. However, suppose we consider the processor-sharing discipline: when $\left|S_{t}\right|>0$ every occupied location is being served, but at a rate $\mu /\left|S_{t}\right|$, so that the overall departure rate from a nonempty system is still $\mu$. In this case, $\left[S_{t} \cap\{1,2, \cdots, m\}, S_{t} \cap\{m+1, m+2, \cdots\}\right]$ retains the Markov property, but unfortunately, the partial differential equation for the generating function appears to be quite intractable. It is again apparent that one must resort to a numerical study.

Acknowledgments. We are grateful to J. C. Lagarias for assistance in obtaining the bound (2.12), and J. A. Morrison for interest and discussions on the asymptotics of the formula (2.3) as $\rho \rightarrow \infty$.

\section{REFERENCES}

[1] E. G. CoffmAn, JR., An introduction to combinatorial models of dynamic storage allocation, SIAM Rev., 25 (1983), pp. 311-325. 
[2] E. G. Coffman, JR., T. T. Kadota And L. A. Shepp, On the asymptotic optimality of first-fit storage allocation, IEEE Trans. Software Engineering, to appear.

[3] E. G. Coffman, JR. AND A. C. MCKellar, On the motion of an unbounded, Markov queue in random access storage, IEEE Trans. Comput., June, 1968.

[4] W. Feller, An Introduction to Probability Theory and Its Applications, Vol. 1, 2nd Edition, John Wiley, New York, 1950.

[5] T. T. Kadota, L. A. ShePp AND J. ZIV, Asymptotic efficiency of dynamic storage systems, to appear.

[6] D. E. KNUTH, Fundamental Algorithms, Vol. 1, 2nd edition, Addison-Wesley, 1973, Reading, MA, Section 2.5.

[7] F. SPITZER, Interaction of Markov processes, Advances in Math, (1970) pp. 246-290. 